\title{
Generation of Multipartite Entangled States Using Switchable Coupling of Cooper-Pair-Boxes
}

\author{
Stefan J. Rinner, Tindaro Pittorino \\ Interstate University of Applied Science NTB, Buchs, Switzerland \\ Email: stefan.rinner@ost.ch
}

How to cite this paper: Rinner, S.J. and Pittorino, T. (2020) Generation of Multipartite Entangled States Using Switchable Coupling of Cooper-Pair-Boxes. Journal of Modern Physics, 11, 1514-1527. https://doi.org/10.4236/jmp.2020.1110093

Received: August 5, 2020

Accepted: October 11, 2020

Published: October 14, 2020

Copyright $\odot 2020$ by author(s) and Scientific Research Publishing Inc. This work is licensed under the Creative Commons Attribution International License (CC BY 4.0).

http://creativecommons.org/licenses/by/4.0/

\section{(c) (i) Open Access}

\begin{abstract}
We propose a scheme for a switchable coupling between several Cooper-pair boxes in the charge regime. The switch is embodied in a SQUID element contained in the center conductor of a transmission-line resonator. Altering the flux bias through the SQUID allows for changing the effective resonator length. Thus the position of the nodes and anti-nodes of the relevant eigenmodes changes and leads to a variable interaction strength between qubit and cavity vacuum field. For the coupled qubits the interaction is dispersive. An example for the application of this switch is the generation of multipartite entangled states for three and four charge qubits. Although used as a discrete switch in the present proposal, the combined system of SQUID module integrated into the transmission line may be operated continuously as well.
\end{abstract}

\section{Keywords}

Switchable, Variable Interaction

\section{Introduction}

Quantum Optics on a chip represents an intriguing and promising pace on the way to a scalable Quantum Computer. In [1] and [2] the strong coupling of a single photon to a superconducting qubit using circuit quantum electrodynamics has been demonstrated and their work was followed by other investigations in this field [3] [4] [5]. Some years ago, the construction of a reconfigurable quantum optical circuit on a chip and the control of entangled states with up to four photons were reported [6]. At the heart of this device is a simple heating element that changes the phase in one arm of an interferometer. In addition, there exist proposals for the design and fabrication of photonic quantum circuits 
[7] as well as their characterization [8]. In [9] researchers report that on a single chip, sources of entangled photons are combined with optical elements that can perform complex manipulations of quantum signals. Other groups have realised quantum circuits using quantum dots and superconducting devices [10]. In this context, mesoscopic devices, such as the Cooper-pair box (CPB), appear to be promising candidates for building blocks in the hardware of a quantum computer. The logic states of the qubit are formed by a large number of Cooper pairs and are distinguished by the number of excess Cooper pairs on the superconducting island which is connected to a superconducting reservoir by a Josephson junction.

Exemplarily, more recent publications [11]-[16] demonstrate that the interest in superconducting qubits for quantum computation still remains a vivid area of research.

Probably the most prominent advantage of mesoscopic systems, like the CPB or quantum dots, compared to atomic systems or photons is the fact that they can be fabricated and operated with standard present-day technology, and that their designs are scalable. In the fabrication process their properties can be customized, and no extra effort has to be taken to keep their number and location fixed. On the other hand, due to their size they are more strongly exposed to noise and decoherence effects than their atomic or photonic counterparts. The time window available for preparation and coherent manipulation of states in the so-called circuit-QED setup was reported by Wallraff et al. [1] who measured a coherence time as long as $500 \mathrm{~ns}$ for a single CPB inside a cavity. Placing the $\mathrm{CPB}$ in a resonator is believed to screen part of the environmental effects. Therefore, this design is-apart from its importance due to the analogy with cavity QED-a prime candidate for the investigation of multi-qubit systems.

In order to work with multi-qubit devices, e.g. for the realization of quantum-information processing tasks, a tunable coupling is desirable. Lately, a high-fidelity quantum processor was built in the form of a two-dimensional array of 54 transmon qubits [17]. In their experiment, each of these qubits is tunably coupled to four nearest neighbours, in a rectangular lattice. The authors claim to have demonstrated quantum supremacy experimentally. For CPBs, a variety of coupling ideas have been proposed (e.g., [18] [19] [20] [21] [22]). Circuit-QED offers an alternative possibility to couple the CPB via the cavity [1] [23]. In this article we propose a scheme for dispersive CPB coupling. The most important feature of our proposal is the fully controllable switch for inter-qubit coupling where no internal manipulations on the qubits themselves (such as changing the gate voltages on each of them simultaneously and synchronically) are needed to start and stop their interaction. This is achieved by controlling only one external parameter (the magnetic flux through the SQUID loop), increasing the experimental feasibility and reducing possible sources for decoherence. Our proposal is based on the setup of the Yale group [1]. The action of the switch makes use of vacuum modes only. 
With the tunable coupling at hand, one may look for applications based on the manipulation of the interaction between the CPBs inside the cavity. A particularly interesting application is the preparation of multipartite entangled states. For example, one may seek to prepare multipartite states representing locally inequivalent classes of entanglement such as the $G H Z$ and the $W$ state [24]. In cavity QED with atoms there are several theoretical proposals for the generation of such states [25] [26], as well as for trapped ions [27]. In Ref. [28], $G H Z$ states with up to six ions could be generated, while in Ref. [29], $W$ states with up to eight ions have been created. There are other proposals for the generation of such states [30] [31] [32] [33] [34] as well as experimental realisations [35]-[42]. One aim of this work is to present a method of preparing similar states in circuit QED with Cooper pair boxes [43].

A similar proposal was made by Wallquist et al. [44], yet in our approach the bus is used indirectly to obtain effective qubit-qubit coupling, rather than directly with the bus.

In order to support the experimental feasibility of our proposal, we exemplarily mention the work of Palacios et al. [45] and Sandberg et al. [46].

The outline of this paper is as follows. In Section 2 we explain the operation of the switch for turning on and off the interaction between the qubits. We then present a way to use this switch for the controlled generation of multipartite entangled states of the qubits (Section 3). We conclude with a discussion of our results and possibilities to extend them in Section 4.

\section{Switchable Resonator}

\subsection{Concept}

In our proposal, the resonator acts as the coupling device between the qubits. It can be used to control the coupling between an on position and an off position (discrete coupling switch). To this end the center conductor of the resonator contains a SQUID loop. The switch is realized by changing the effective length of the center conductor of the transmission line resonator (Figure 1) via changing the flux through the SQUID loop between integer and half integer number of flux quanta.

The on position of the switch corresponds to the full length $L^{(0)}$ of the center conductor (cf. Figure 1). The CPBs are located where the electric field strength has a node (vanishing coupling between CPB and cavity mode, see Figure 1(a)). Note that we refer to the second eigenmode of the cavity

$$
\omega_{2}^{(0)}=2\left(v \pi / L^{(0)}\right)
$$

with the phase velocity $v=1 / \sqrt{l c}$, in close analogy with Ref. [1], with capacitance per length $c$ and inductance per length $l$. As the total current through a SQUID depends on the external flux $\Phi$ applied to the ring, $J_{\text {total }} \propto \cos \left(\frac{e}{\hbar} \Phi\right)$, for the on position of the switch the flux in the loop needs to be equal to an in- 
teger number of the flux quantum $\Phi_{0}=\frac{h}{e}$. It has been shown that in superconducting rings the magnetic flux is quantised in the following manner $\Phi_{n}=n \cdot \frac{h}{2 e}$. The Josephson energy of the junctions in the SQUID device should be chosen large enough in order not to interrupt the resonator.

If the switch is "off" (i.e., if the flux through the SQUID loop equals a half integer number of flux quanta) the resonator is cut into left and right parts of length $L^{(1)}$ and $L^{(2)}$ respectively: charge cannot flow from the left to the right resonator (see Figure 1(b)). Note however that the electromagnetic field in the left resonator is still coupled capacitively to the rest of the transmission line (through the junction capacitances of the SQUID loop). The CPBs now couple to the second eigenmode of the left resonator as their position is chosen such that the electric field strength is large. In contrast to the setup in Ref. [1] the level splitting of the CPBs is not in resonance with the new eigenfrequency of the left resonator, rather we choose it to be slightly detuned. This causes the qubits to interact with the left cavity mode dispersively without exchanging excitations.

Assuming dimensions in the $\mathrm{cm}$ range for the resonator length and $\sim 2 \mu \mathrm{m}$ for each $\mathrm{CPB}$, an inter- $\mathrm{CPB}$ distance of $\sim 100 \mu \mathrm{m}$ should be sufficient to exclude direct coupling between the $\mathrm{CPBs}$ and, on the other hand, facilitate equal coupling constants of the CPBs with the cavity mode [47].

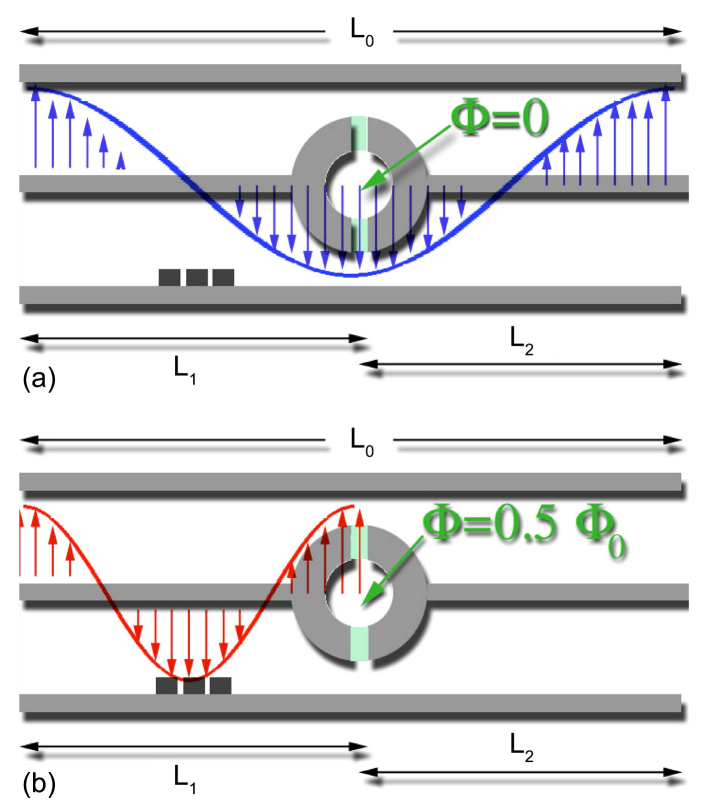

Figure 1. Schematic layout showing three cooper pair boxes (black squares) in the transmission line resonator and the corresponding field modes. (a) Switch in the on position, with SQUID loop closed. The effective resonator length equals the total length $L^{(0)}$ of the center conductor. The $\mathrm{CPB}$ are located at a node position of the $\omega_{2}^{(0)}$ mode; (b) Switch in the off position. The Josephson coupling between left and right part of the center conductor is zero. The new effective resonator length is close to $L^{(0)} / 2$ (see text) and the resonance frequency is detuned from the CPBs' level splitting. 
Let us now consider the effect of the SQUID loop on the transmission line. In general, an infinitesimally short transmission line of length $d x$ can be approximated using the lumped component model depicted in Figure 2.

In this model, the current $I$ and the voltage $U$ are generally functions of the time $t$ and of the position $x$. The values $1, c, r$ and $g$ represent respectively the inductance, the capacitance, the resistance and the conductance per unit of length for the transmission line. Using Kirchoff's voltage and current laws on the lumped model and assuming $d x \rightarrow 0$ the following equations can be derived:

$$
\left\{\begin{array}{l}
\frac{\partial I}{\partial x}=g U+c \frac{\partial U}{\partial t} \\
\frac{\partial U}{\partial x}=r I+l \frac{\partial I}{\partial t}
\end{array}\right.
$$

By calculating the derivative with respect to $x$ of the first equation and the time derivative of the second equation, the current flowing in the transmission line can be expressed by the following expression:

$$
\frac{\partial^{2} I}{\partial x^{2}}=g r I+(g l+r c) \frac{\partial I}{\partial t}+l c \frac{\partial^{2} I}{\partial t^{2}}
$$

In the specific case of a SQUID, the system will only work at very low temperature. Under this condition, it is not false to consider the transmission line as lossless ( $r=0$ and $g=0$ ). Equation (3) can be hence approximated as:

$$
\frac{\partial^{2} i}{\partial x^{2}}=l c \frac{\partial^{2} i}{\partial t^{2}}
$$

Let us consider the effect of the SQUID loop for the resonance frequencies of the transmission line more quantitatively. In an infinitesimal lumped-element circuit model for a transmission line (without SQUID element) the current $I$ obeys the wave equation

$$
\frac{1}{c} \frac{\partial^{2} I}{\partial x^{2}}-l \frac{\partial^{2} I}{\partial t^{2}}=0
$$

with boundary conditions $I(x=0)=I\left(x=L^{(0)}\right)=0$. We assume that this wave equation is satisfied also with the switch in the on position.

For the off position of the switch this equation is modified. While in the left and the right part of the transmission line Equation (5) remains unchanged, at

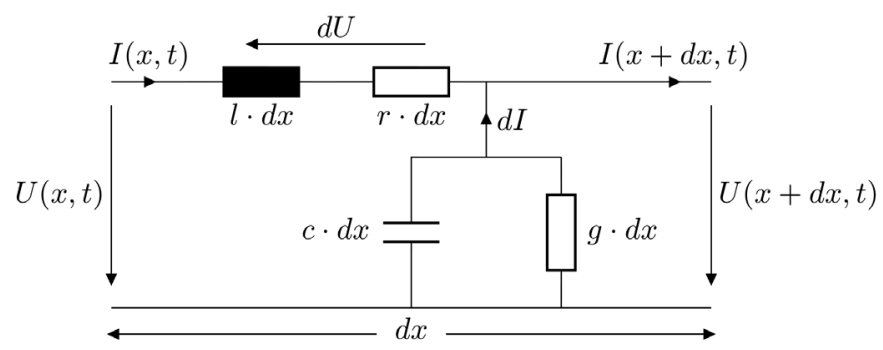

Figure 2. Transmission line lumped model for an infinitesimally short line. 
$x=x_{0}$ the total capacitance $C_{0}$ of the junctions in the loop (which can be assumed to be point-like at the position $x_{0}$ ) contributes another term that couples left and right resonator part:

$$
\frac{1}{c} \frac{\partial^{2} I}{\partial x^{2}}-l \frac{\partial^{2} I}{\partial t^{2}}-\frac{1}{C_{0}} I \delta\left(x-x_{0}\right)=0 .
$$

The resulting problem for the eigenmodes of this resonator is analogous to a quantum-mechanical particle of mass $c / 2$ in a double-well potential (of lengths $L^{(1)}$ and $\left.L^{(2)}\right)$ separated by a $\delta$-barrier of strength $1 / C_{0}$ at $x=x_{0}$. We choose $L^{(1)}, L^{(2)}$ to differ by a few per cent in order to avoid the symmetry point $L^{(1)}=L^{(2)}$ for which the solutions have peculiar properties. Moreover, this allows us to arrange for the proper detunings of the various modes. We are interested in the case of weak coupling between the "potential wells", that is, weak capacitive coupling $C_{0} \ll c L^{(0)}$ between the parts of the center conductor. In this case the new eigenmodes differ only little from the modes of the unperturbed problem (that is, no coupling at all, $C_{0} \rightarrow 0$ ). To first order we obtain, e.g., for the second mode of the left resonator (cf. also Ref. [1])

$$
\omega_{2}^{(1)} \approx 2 \frac{v \pi}{L^{(1)}}\left(1-\frac{C_{0}}{c L^{(1)}}\right)
$$

The first term in this expression represents the resonant solution (cf. Equation (1)), the second one accounts for the effect of detuning. Given the typical capacitance of a transmission line, the capacitance $C_{0}$ may be on the order of several $10^{-14} \mathrm{~F}$ to satisfy the weak-coupling condition.

\subsection{Quantitative Considerations}

In this section we briefly discuss the relevant modes for the two regimes of the switch and estimate possible errors. When the cavity mode is in resonance with the qubit frequency there is coupling between cavity and CPBs with coupling strength $g$ which is proportional to the strength of the local electric field, i.e. the coupling with resonant modes vanishes at their nodes and is maximal at their antinodes. This coupling strength is modified in the case of detuning and results in an effective coupling strength $\lambda_{i}^{k}=g^{2} / \Delta_{i}^{(k)}$ as will be shown in the following paragraph.

In the off regime for modes $\omega_{i}^{(1)}$ in the left resonator $\Delta_{i}^{(1)}$ is large (on the order of the cavity eigenfrequency) only except for the slightly detuned $\omega_{2}^{(1)}$. The mode $\omega_{2}^{(2)}$ (eigenmode of the right resonator) might leak into the left part if the disconnection is not perfect. However, not only is it also detuned (given $L_{1} \neq L_{2}$ ) but also decays its amplitude rapidly at the left side rendering the coupling to this mode a negligible quantity at the site of the CPBs.

In the on regime the CPBs are sited at a node of the (also detuned) mode $\omega_{2}^{(0)}$, so that the coupling vanishes. The mode with the smallest detuning with respect to the $\mathrm{CPB}$ eigenfrequency in this regime is $\omega_{4}^{(0)}$. Its impact on the $\mathrm{CPB}$ may still be ignored as discussed in further detail below. 
In order to realize a dispersive interaction of the CPBs with cavity mode $\omega_{2}^{(1)}$, we require a detuning of, say $\delta \omega_{2}^{(1)} / \omega_{2}^{(1)} \sim 10^{-2}$. The other resonator modes need to have much stronger detuning in order to reduce their coupling to the qubit, in particular for the on position of the switch. For example, we may choose $L^{(1)} \simeq 0.54 L^{(0)}$ and $C_{0} / c L^{(1)} \simeq 0.05$ which yields $\sim 10 \%$ red shift of the resonance frequency $\omega_{2}^{(0)}$ from the closest mode of the off switch and $\sim 12 \%$ detuning from the 4th mode of the full-length resonator. For coupling strengths in the $\mathrm{MHz}$ regime and frequencies on the order of $10^{10} \mathrm{~Hz}$ this detuning leads to an effective coupling strength $\lambda \simeq 10^{-3} \mathrm{~g}$ with respect to the coupling strength $g$ in the resonant case.

A CPB spacing of $\Delta x=100 \mu \mathrm{m}$ corresponds to one per cent of the $\mathrm{cm} \mathrm{di-}$ mensions of the resonator. Consequently, the amplitude of the mode $\omega_{2}^{(1)}$ differs from its maximal value at position $x_{\max }$ by a factor of around $10^{-4}$ at positions $x=x_{\max } \pm 100 \mu \mathrm{m}$ and by a factor of $10^{-3}$ for $\Delta x=300 \mu \mathrm{m}$ for a resonator length of $3 \mathrm{~cm}$.

In the following section we will show how to use this digital switch in order to generate multipartite entanglement between the qubits via the dispersive interaction with the cavity mode.

\section{Generation of Multipartite Entangled States}

The starting configuration of the system cavity plus CPBs is the following: the flux through the ring configuration is $\Phi=0$. The state of the cavity field is assumed to be the vacuum state $\left|0_{f}\right\rangle_{0}$ where the first subscript stands for "field" and the second denotes the cavity eigenmode of the resonator with length $L^{(0)}$.

In the interaction picture the system is described by the Tavis-Cummings Hamiltonian [48], the $N$-atom generalized Jaynes-Cummings Hamiltonian [49]:

$$
H_{0}=\hbar g_{0} \sum_{j=1,2,3}\left(\mathrm{e}^{-i \delta t} a^{\dagger} \sigma_{j}^{-}+\mathrm{e}^{i \delta t} a \sigma_{j}^{+}\right)
$$

containing the bosonic creation (annihilation) operators $a^{\dagger}(a)$ for a photon in the cavity mode, the creation (annihilation) operators $\sigma_{j}^{\dagger}\left(\sigma_{j}\right)$ for an excitation of the $j$ th Cooper pair box, the detuning $\delta$ and the coupling strength $g_{0}$ between the dipole moment of each CPB and the electric field. In the interest of legibility we use $g_{0}$ instead of $g_{2}^{(0)}$. The three CPBs are assembled at a position where the electric field strength of the relevant cavity eigenmode vanishes. Consequently, there is (approximately) no interaction beyond the free evolution $\left(g_{0} \simeq 0\right.$ ). Due to this condition a well-defined initial state can be prepared that does not exchange excitations with the cavity field.

Suppose now the flux through the SQUID is suddenly changed by half a flux quantum. The effective resonator length changes to the smaller $L^{(1)}$ with the new eigenmode in its vacuum state $\left|0_{f}\right\rangle_{L}$. The new resonator frequency $\omega_{2}^{(1)}$ is detuned from the $\mathrm{CPB}$ resonance and the coupling strength $g_{L}$ (again instead of $g_{2}^{(1)}$ ) still being near its maximal value. Denoting the detuning between cavity mode and each CPB by $\Delta$ and the creation (annihilation) operators for 
the cavity mode b by $b^{\dagger}(b)$, the new Tavis-Cummings Hamiltonian can be written in the interaction picture as

$$
H=\hbar g_{L} \sum_{j=1,2,3}\left(\mathrm{e}^{-i \Delta t} b^{\dagger} \sigma_{j}^{-}+\mathrm{e}^{i \Delta t} b \sigma_{j}^{+}\right) .
$$

The corresponding time-evolution operator $U(t)=U\left(t, t_{0}=0\right)$ is given up to second order by

$$
U(t) \approx 1-\frac{i}{\hbar} \int_{0}^{t} \mathrm{~d} t^{\prime} H\left(t^{\prime}\right)-\frac{1}{\hbar^{2}} \int_{0}^{t} \mathrm{~d} t^{\prime} H\left(t^{\prime}\right) \int_{0}^{t^{\prime}} \mathrm{d} t^{\prime \prime} H\left(t^{\prime \prime}\right) .
$$

We use this expansion to derive the effective Hamiltonian for large detuning, i.e., $\Delta \gg g_{L}$, where no energy exchange between the CPB system and the cavity is possible. The first-order term gives

$$
\begin{aligned}
U^{(1)}(t) & =-\frac{i}{\hbar} \int_{0}^{t} \mathrm{~d} t^{\prime} H\left(t^{\prime}\right) \\
& =\frac{g_{L}}{\Delta} \sum_{j=1,2,3}\left[\left(\mathrm{e}^{-i \Delta t}-1\right) b^{\dagger} \sigma_{j}^{-}-\left(\mathrm{e}^{i \Delta t}-1\right) b \sigma_{j}^{+}\right],
\end{aligned}
$$

and the second-order term

$$
\begin{aligned}
U^{(2)}(t) & =-\frac{1}{\hbar^{2}} \int_{0}^{t} \mathrm{~d} t^{\prime} H\left(t^{\prime}\right) \int_{0}^{t^{\prime}} \mathrm{d} t^{\prime \prime} H\left(t^{\prime \prime}\right) \\
& =-\frac{i g_{L}^{2}}{\Delta} \int_{0}^{t} \mathrm{~d} t^{\prime} \sum_{j=1}^{3}\left(\mathrm{e}^{-i \Delta t^{\prime}} b^{\dagger} \sigma_{j}^{-}+\mathrm{e}^{i \Delta t^{\prime}} b \sigma_{j}^{+}\right) \sum_{k=1}^{3}\left(\left(\mathrm{e}^{-i \Delta t^{\prime}}-1\right) b^{\dagger} \sigma_{j}^{-}-\left(\mathrm{e}^{i \Delta t^{\prime}}-1\right) b \sigma_{j}^{+}\right) .
\end{aligned}
$$

Note that after carrying out the above product of sums the subsequent integration will yield additional prefactors of order $1 / \Delta$ for all terms except for those which involve the product of complex conjugate exponentials. With this in mind it is easy to see that

$$
\begin{aligned}
U^{(2)}(t)= & -\frac{i g_{L}^{2}}{\Delta} \sum_{j=1}^{3}\left(-b^{\dagger} b \sigma_{j}^{-} \sigma_{j}^{+}+b b^{\dagger} \sigma_{j}^{+} \sigma_{j}^{-}\right) t \\
& -\frac{i g_{L}^{2}}{\Delta} \underbrace{\left(-b^{\dagger} b+b b^{\dagger}\right)}_{=\left[b, b^{\dagger}\right]=1}\left(\sigma_{1}^{-} \sigma_{2}^{+}+\sigma_{1}^{-} \sigma_{3}^{+}+\sigma_{2}^{-} \sigma_{1}^{+}\right. \\
& \left.+\sigma_{2}^{-} \sigma_{3}^{+}+\sigma_{1}^{+} \sigma_{3}^{-}+\sigma_{2}^{+} \sigma_{3}^{-}\right) t+\mathcal{O}\left(\frac{1}{\Delta^{2}}\right) .
\end{aligned}
$$

Here and in the following $\sigma_{1}^{-} \sigma_{3}^{+}$, e.g., is shorthand for $\sigma_{1}^{-} \otimes 1_{2} \otimes \sigma_{3}^{+}$. Keeping only terms linear in time we arrive at

$$
\begin{aligned}
U(t, 0) \approx & 1-\frac{i}{\hbar}\left(\frac{\hbar g_{L}^{2}}{\Delta}\right)\left[\sum_{j=1}^{3}\left(b b^{\dagger} \sigma_{j}^{+} \sigma_{j}^{-}-b^{\dagger} b \sigma_{j}^{-} \sigma_{j}^{+}\right)\right. \\
& \left.+\left(\sigma_{2}^{-} \sigma_{3}^{+}+\sigma_{1}^{-} \sigma_{3}^{+}+\sigma_{1}^{+} \sigma_{2}^{-}+\sigma_{1}^{+} \sigma_{3}^{-}+\sigma_{2}^{+} \sigma_{3}^{-}+\sigma_{2}^{+} \sigma_{1}^{-}\right)\right] t \\
\approx & \exp \left[-\frac{i}{\hbar} H_{\text {eff }} t\right] .
\end{aligned}
$$

with the effective Hamiltonian in the interaction picture 


$$
\begin{aligned}
H_{\mathrm{eff}}= & \hbar \underbrace{\left(\frac{g_{L}^{2}}{\Delta}\right)}_{=: \lambda}\left[\sum_{j=1,2,3}\left(b b^{\dagger} \sigma_{j}^{+} \sigma_{j}^{-}-b^{\dagger} b \sigma_{j}^{-} \sigma_{j}^{+}\right)+\left(\sigma_{2}^{-} \sigma_{3}^{+}+\sigma_{1}^{-} \sigma_{3}^{+}\right.\right. \\
& \left.\left.+\sigma_{1}^{+} \sigma_{2}^{-}+\sigma_{1}^{+} \sigma_{3}^{-}+\sigma_{2}^{+} \sigma_{3}^{-}+\sigma_{2}^{+} \sigma_{1}^{-}\right)\right] .
\end{aligned}
$$

Consider now the case when the cavity is in its vacuum state. Then the above Hamiltonian reduces to

$$
H_{\mathrm{eff}}=\hbar \lambda\left[\sum_{j=1,2,3}\left|e_{j}\right\rangle\left\langle e_{j}\right|+\left(\sigma_{2}^{-} \sigma_{3}^{+}+\sigma_{1}^{-} \sigma_{3}^{+}+\sigma_{1}^{+} \sigma_{2}^{-}+\sigma_{1}^{+} \sigma_{3}^{-}+\sigma_{2}^{+} \sigma_{3}^{-}+\sigma_{2}^{+} \sigma_{1}^{-}\right)\right] \text {, }
$$

since $b b^{\dagger}\left|0_{f}\right\rangle_{L}=\left|0_{f}\right\rangle_{L}, b^{\dagger} b\left|0_{f}\right\rangle_{L}=0$.

This shows that there is an effective interaction between the qubits even though no excitation is transferred from the qubits to the cavity mode. Next we want to show how to use such interactions in order to generate three-qubit and four-qubit entangled states. To this end, one has to solve the Schrödinger equation for this Hamiltonian. In the following, applying the conventional nomenclature for qubits let $\left|1_{j}\right\rangle$ denote the excited state of the jth CPB $\left|e_{j}\right\rangle$ since no field Fock states are needed anymore and confusion is thus avoided. States which initially are computational basis states can be divided into three classes with the corresponding time evolution:

1) only one CPB is in state $|1\rangle$, e.g., $|\psi(0)\rangle=|100\rangle$ :

$$
\left|\psi_{1}(t)\right\rangle=\frac{\mathrm{e}^{-3 i \lambda t}+2}{3}|100\rangle+\frac{\mathrm{e}^{-3 i \lambda t}-1}{3}(|010\rangle+|001\rangle) .
$$

2) only one $\mathrm{CPB}$ is in state $|0\rangle$, e.g. $|\psi(0)\rangle=|110\rangle$ :

$$
\left|\psi_{2}(t)\right\rangle=\frac{1}{3} \mathrm{e}^{-i \lambda t}\left[\left(\mathrm{e}^{-3 i \lambda t}+2\right)|110\rangle+\left(\mathrm{e}^{-3 i \lambda t}-1\right)(|011\rangle+|101\rangle)\right] .
$$

3) all CPBs are in the same state, i.e. $\left|\psi_{3}(t)\right\rangle=|000\rangle$ or $\left|\psi_{3}(t)\right\rangle=|111\rangle$.

With the coupling switched off, the state of the CPB system only acquires a phase factor (we assume that, in principle, such states can be prepared). Now the dispersive coupling is switched on suddenly. By starting from the state of the first type and adjusting the interaction time such that $\tau=\frac{2 \pi}{9 \lambda}$ the state of the $\mathrm{CPB}$ system evolves into a $W$ state of the form

$$
\begin{aligned}
\left|\psi_{1}(\tau)\right\rangle & =\frac{\mathrm{e}^{-i 2 \pi / 3}+2}{3}|100\rangle+\frac{\mathrm{e}^{-i 2 \pi / 3}-1}{3}(|010\rangle+|001\rangle) \\
& =\frac{1}{\sqrt{3}}\left[\mathrm{e}^{-i \pi / 6}|100\rangle+\mathrm{e}^{-i 5 \pi / 6}(|010\rangle+|001\rangle)\right] \\
& =\mathrm{e}^{-i 5 \pi / 6} \frac{1}{\sqrt{3}}\left[\mathrm{e}^{i 2 \pi / 3}|100\rangle+(|010\rangle+|001\rangle)\right] .
\end{aligned}
$$

For a detuning $\Delta \sim 100 \mathrm{MHz}$ and a coupling strength $g_{L} \sim 15 \mathrm{MHz}$, the time required to generate this $W$ state is $\tau \sim 300 \mathrm{~ns}$.

This is an example of the general $N$-qubit $W$ state with arbitrary phases: 


$$
\begin{aligned}
\left|W_{N}\right\rangle= & \frac{1}{\sqrt{N}}\left(\mathrm{e}^{i \theta_{1}}|1000 \cdots 0\rangle+\mathrm{e}^{i \theta_{2}}|0100 \cdots 0\rangle+\mathrm{e}^{i \theta_{3}}|0010 \cdots 0\rangle+\cdots\right. \\
& \left.+\mathrm{e}^{i \theta_{N}}|0000 \cdots 1\rangle\right)
\end{aligned}
$$

The concurrences between any two qubits of the above state are all equal to $2 / N$ and do not depend on the phases [50].

By extending this method to four qubits it is also possible to generate a three-CPB $W$ state with equal phases: Starting from one CPB in the excited state and the remaining three CPBs in the ground state $\left|\psi_{4}(t=0)\right\rangle=|1000\rangle$ the dispersive interaction leads to a state

$$
\left|\psi_{4}(t)\right\rangle=c_{1}(t)|1000\rangle+c_{2}(t)[|0100\rangle+|0010\rangle+|0001\rangle]
$$

with the probability amplitudes

$$
\begin{gathered}
c_{1}(t)=\frac{\sqrt{3}+1}{2 \sqrt{3}} \mathrm{e}^{i(\sqrt{3}-2) \lambda t}+\frac{\sqrt{3}-1}{2 \sqrt{3}} \mathrm{e}^{-i(\sqrt{3}+2) \lambda t} \\
c_{2}(t)=\frac{1}{2 \sqrt{3}}\left(\mathrm{e}^{-i(\sqrt{3}+2) \lambda t}-\mathrm{e}^{i(\sqrt{3}-2) \lambda t}\right) .
\end{gathered}
$$

Detection of the first qubit in the state $|0\rangle$ then gives the desired $W$ state for the remaining three CPBs:

$$
|W(t)\rangle=\frac{1}{\sqrt{3}}|0\rangle \otimes[|100\rangle+|010\rangle+|001\rangle]
$$

where the common phase factor has been discarded.

We emphasize that there is no need for a specific interaction time in order to create this superposition state. The trade-off, however, is in the necessity to perform a read-out on the first qubit and the probabilistic nature of the preparation procedure.

As a final example we mention the generation of $G H Z$-like states for four qubits. By choosing the initial state $\left|\psi_{5}(t=0)\right\rangle=|1100\rangle$ the time evolution with the interaction switched on results in

$$
\left|\psi_{5}(t)\right\rangle=C_{1}(t)|1100\rangle+C_{2}(t)|0011\rangle+C_{3}(t)[|1010\rangle+|1001\rangle+|0110\rangle+|0101\rangle]
$$

with probability amplitudes

$$
\begin{gathered}
C_{1}(t)=\frac{1}{6} \mathrm{e}^{-4 i \lambda t}+\frac{1}{3} \mathrm{e}^{2 i \lambda t}+\frac{1}{2} \\
C_{2}(t)=\frac{1}{6} \mathrm{e}^{-4 i \lambda t}+\frac{1}{3} \mathrm{e}^{2 i \lambda t}-\frac{1}{2} \\
C_{3}(t)=\frac{1}{6} \mathrm{e}^{-4 i \lambda t}-\frac{1}{6} \mathrm{e}^{2 i \lambda t}
\end{gathered}
$$

Noting that $C_{3}(\tau)=0$ for the choice $\tau=\pi / 3 \lambda$ one can prepare a state belonging to the $G H Z$-class [24] [26] [51] of the form

$$
\left|\psi_{5}(\tau)\right\rangle=\frac{1}{4}[(i \sqrt{3}+1)|1100\rangle+(i \sqrt{3}-3)|0011\rangle]
$$

as desired. 


\section{Conclusions}

We have presented a theoretical proposal to implement a switch for controlled dispersive coupling of several Cooper pair boxes in a transmission line resonator. The coupling can be turned on and off by changing the flux through a SQUID loop integrated into the center conductor of the resonator. Here a few additional remarks are due regarding the practical realization of this idea.

First, we have discussed the switch as a digital device between an "on" and an "off" position. The latter position is, strictly speaking, only an "almost off" position with a much smaller coupling $g_{0} \ll g_{L}$ compared to the "on" case. This is due to the structure of the effective coupling constant $g_{L}^{2} / \Delta$ : due to the presence of the other modes (far off resonance) there is always a residual coupling. As we have mentioned in Section 2, a difference of at least one order of magnitude is realistic. This sets a limit for the idle periods of the setup: if this limit is exceeded, the time evolution of the (almost) uncoupled CPB system is not proportional to the identity (apart from a phase factor), but a more general uncontrolled many-qubit gate.

Secondly, the switching operation is not limited to the digital mode. In principle, the resonator modes constitute continuous functions of the flux $\Phi$ through the SQUID loop. As the discussion in Section 2 shows, one may view the action of the loop also as a control of the boundary conditions for the electromagnetic field in the transmission line (see also a related proposal [52]). A detailed analysis needs to take into account Josephson inductance $L_{J}$ of the SQUID loop that depends on the (flux-dependent) Josephson energy $E_{J}(\Phi)$ as $L_{J} \propto 1 / E_{J}(\Phi)$ in parallel to the capacitance $C_{0}$, and will be carried out elsewhere.

Apart from the advantages of the switch (e.g., no simultaneous switching of $\mathrm{CPB}$ controls) that contribute to reducing noise, the presence of the SQUID loop introduces also new decoherence sources: the Josephson junctions of the loop (which are subject to dielectric losses [53]) and flux noise.

Finally, we have discussed the application of this coupling scheme for the generation of multi-qubit entanglement between the CPBs. We have emphasized the principle of the idea, and we have not considered a detailed setup which should include also the methods for state preparation and measurements. In particular, we have shown how $W$ states for three qubits and $G H Z$ states for four qubits can be obtained. The protocols are surprisingly simple and, apart from state preparation and measurements, do not require complicated sequences of operations and can be realized in a single shot. Thus one may conclude that the setup provides an interesting starting point for various multi-qubit applications based on circuit-QED.

\section{Acknowledgements}

We would like to thank G. Falci and E. Werner for illuminating discussions and comments. 


\section{Conflicts of Interest}

The authors declare no conflicts of interest regarding the publication of this paper.

\section{References}

[1] Blais, A., Huang, R.-S., Wallraff, A., Girvin, S.M. and Schoelkopf, R.J. (2004) Physical Review A, 69, Article ID: 062320. https://doi.org/10.1103/PhysRevA.69.062320

[2] Wallraff, A., Schuster, D.I., Blais, A., Franzio, L., Huang, R.-S., Majer, J., Kumar, S., Girvin, A.M. and Schoelkopf, R.J. (2004) Nature, 431, 162-167. https://doi.org/10.1038/nature02851

[3] Irish, E.K., Gea-Banaloche, J., Martin, I. and Schwab, K.C. (2005) Physical Review B, 72, Article ID: 195410. https://doi.org/10.1103/PhysRevB.72.195410

[4] Devoret, M.H., Girvin, S. and Schoelkopf, R. (2007) Annals of Physics, 16, 767. https://doi.org/10.1002/andp.200710261

[5] You, J.Q., Liu, Y.X., Sun, C.P. and Nori, F. (2007) Physical Review B, 75, Article ID: 104516. https://doi.org/10.1103/PhysRevB.75.104516

[6] Berr, D.W. and Wiseman, H.M. (2009) Nature Photonics, 3, 317-319. https://doi.org/10.1038/nphoton.2009.84

[7] Poot, M., Schuck, C., Ma, X.-S., Guo, X. and Tang, H.X. (2016) Optics Express, 24, 6843-6860. https://doi.org/10.1364/OE.24.006843

[8] Poot, M. and Tang, H.X. (2016) Applied Physics Letters, 109, Article ID: 131106. https://doi.org/10.1063/1.4962902

[9] Jin, H., Liu, F.M., Xu, P., Xia, J.L., Zhong, M.L., Yuan, Y., Zhou, J.W., Gong, Y.X., Wang, W. and Zhu, S.N. (2014) Physical Review Letters, 113, Article ID: 103601. https://doi.org/10.1103/PhysRevLett.113.103601

[10] Deng, G.-W., Guo, G.-P. and Guo, G.-C. (2016) On-Chip Quantum Optics with Quantum Dots and Superconducting Resonators. Proc. SPIEE 10029, Quantum and Nonlinear Optics, Vol. 4, Article ID: 1002909.

[11] Liu, Y.-X., Xu, X.-W., Miranowicz, A. and Nori, F. (2014) Physical Review A, 89, Article ID: 043818. https://doi.org/10.1103/PhysRevA.89.043818

[12] Mirza, I.M. and Schotland, J.C. (2016) Physical Review A, 94, Article ID: 012309. https://doi.org/10.1103/PhysRevA.94.012302

[13] Armata, F., Calajo, G., Jaako, T., Kim, M.-S. and Rabl, P. (2017) Physical Review Letters, 119, Article ID: 183602. https://doi.org/10.1103/PhysRevLett.119.183602

[14] Blais, A., Girvin, S.M. and Oliver, W.D. (2020) Nature Physics, 16, 247-256. https://doi.org/10.1038/s41567-020-0806-Z

[15] Mirza, I.M. and Schotland, J.C. (2016) Physical Review A, 94, Article ID: 012302. https://doi.org/10.1103/PhysRevA.94.012302

[16] Ciccarello, F., Paternostro, M., Palma, G.M. and Zarcone, M. (2009) New Journal of Physics, 11, Article ID: 073040. https://doi.org/10.1088/1367-2630/11/11/113053

[17] Arute, F., et al. (2019) Nature, 574, 505-510.

[18] Makhlin, Yu., Shnirman, S. and Schön, G. (1999) Nature, 398, 305-307.

[19] Siewert, J., Fazio, R., Palma, G.M. and Sciacca, E. (2000) Journal of Low Temperature Physics, 18, 795. https://doi.org/10.1023/A:1004612016347

[20] You, J.Q., Tsai, J.S. and Nori, F. (2002) Physical Review Letters, 89, Article ID: 197902. https://doi.org/10.1103/PhysRevLett.89.197902 
[21] Averin, D.V. and Bruder, C. (2003) Physical Review Letters, 91, Article ID: 057003. https://doi.org/10.1103/PhysRevLett.91.057003

[22] Lantz, J., Wallquist, M., Shumeiko, V.S. and Wendin, G. (2004) Physical Review B, 70, Article ID: 140507(R). https://doi.org/10.1103/PhysRevB.70.140507

[23] Plastina, F. and Falci, G. (2003) Physical Review B, 67, Article ID: 224514. https://doi.org/10.1103/PhysRevB.67.224514

[24] Dür, W., Vidal, G. and Cirac, J.I. (2000) Physical Review A, 62, Article ID: 062314. https://doi.org/10.1103/PhysRevA.62.062314

[25] Zeilinger, A., Horne, M.A. and Greenberger, D.M. (1997) NASA Conference Publication No. 3135. National Aeronautics and Space Administration, Code NTT, Washington DC.

Guo, G.-C. and Zhang, Y.-S. (2002) Physical Review A, 65, Article ID: 054302.

[26] Guo, G.-P., Li, C.-F., Li, J. and Guo, G.-C. (2002) Physical Review A, 65, Article ID: 042102. https://doi.org/10.1103/PhysRevA.65.042102

[27] Roos, C.F., Riebe, M., Häffner, H., Hänsel, W., Benhelm, J., Lancaster, G.P.T., Becher, C., Schmidt-Kaler, F. and Blatt, R. (2004) Science, 304, 1478.

[28] Leibfried, D., Knill, E., Seidelin, S., Britton, J., Blakestad, R.B., Chiaverini, J., Hume, D.B., Itano, W.M., Jost, J.D., Langer, C., Ozeri, R., Reichle, R. and Wineland, D.J. (2005) Nature, 438, 639. https://doi.org/10.1038/nature04251

[29] Häffner, H., Hänsel, W., Roos, C.F., Benhelm, J., Chek-al-kar, D., Chwalla, M., Körber, T., Rapol, U.D., Riebe, M., Schmidt, P.O., Becher, C., Gühne, O., Dür, W. and Blatt, R. (2005) Nature, 438, 643. https://doi.org/10.1038/nature04279

[30] Peng, Z.H., Liu, Y.-X., Nakamura, Y. and Tsai, J.S. (2012) Physical Review B, 85, Article ID: 024537. https://doi.org/10.1103/PhysRevB.85.024537

[31] Yang, C.-P. (2011) Physical Review A, 83, Article ID: 062302. https://doi.org/10.1103/PhysRevA.83.042112

[32] Wang, Y.-D., Chesi, S., Loss, D. and Bruder, C. (2010) Physical Review B, 81, Article ID: 104524. https://doi.org/10.1103/PhysRevB.81.104524

[33] Aldana, S., Wang, Y.-D. and Bruder, C. (2011) Physical Review B, 84, Article ID: 134519. https://doi.org/10.1103/PhysRevB.84.134519

[34] Bishop, L.S., et al. (2009) New Journal of Physics, 11, Article ID: 073040.

[35] Song, C., Xu, K., Liu, W.X., Yang, C.-P., Zheng, S.-B., Deng, H., Xie, Q.W., Huang, K.Q., Guo, Q.J., Zhang, L.B., Zhang, P.F., Xu, D., Zheng, D.N., Zhu, X.B., Wang, H., Chen, Y.-A., Lu, C.-Y., Han, S.Y. and Pan, J.-W. (2017) Physical Review Letters, 119, Article ID: 180511. https://doi.org/10.1103/PhysRevLett.119.180511

[36] Barends, R., Kelly, J., Megrant, A., et al. (2014) Nature, 508, 500-503. https://doi.org/10.1038/nature13171

[37] Huang, S.-Y., Goan, H.-S., Li, X.-Q. and Milburn, G.J. (2013) Physical Review A, 88, Article ID: 062311. https://doi.org/10.1103/PhysRevA.88.062311

[38] Feng, W., Wang, P.Y., Ding, X.M., Xu, L.T. and Li, X.-Q. (2011) Physical Review A, 83, Article ID: 042313.

[39] Di Carlo, L., Reed, M., Sun, L., et al. (2010) Nature, 467, 574-578. https://doi.org/10.1038/nature09416

[40] Neeley, M., Bialczak, R., Lenander, M., et al. (2010) Nature, 467, 570-573. https://doi.org/10.1038/nature09418

[41] Chow, J.M., DiCarlo, L., Gambetta, J.M., Nunnenkamp, A., Bishop, L.S., Frunzio, L., Devoret, M.H., Girvin, S.M. and Schoelkopf, R.J. (2010) Physical Review A, 81, Ar- 
ticle ID: 062325. https://doi.org/10.1103/PhysRevA.81.062325

[42] Majer, J., Chow, J., Gambetta, J., et al. (2007) Nature, 449, 443-447. https://doi.org/10.1038/nature06184

[43] Guillaume, A. and Dowling, J.P. (2006) Physical Review A, 73, Article ID: 040304(R).

[44] Wallquist, M., Shumeiko, V.S. and Wendin, G. (2006) Physical Review B, 74, Article ID: 224506. https://doi.org/10.1103/PhysRevB.74.224506

[45] Palacios-Laloy, A., Nguyen, F., Mallet, F., et al. (2008) Journal of Low Temperature Physics, 151, 1034-1042. https://doi.org/10.1007/s10909-008-9774-X

[46] Sandberg, M., et al. (2008) Applied Physics Letters, 92, Article ID: 203501. https://doi.org/10.1063/1.2929367

[47] Wallraff, A. (2005) Private Communication.

[48] Tavis, M. and Cummings, F.W. (1968) Physical Review, 170, 379. https://doi.org/10.1103/PhysRev.170.379

[49] Jaynes, E.T. and Cummings, F.W. (1963) Proceedings of the IEEE, 51, 89. https://doi.org/10.1109/PROC.1963.1664

[50] Wang, X. (2001) Physical Review A, 64, Article ID: 012313. https://doi.org/10.1103/PhysRevA.64.012313

[51] Greenberger, D.M., Horne, M. and Zeilinger, A. (1980) Going beyond Bell's Theorem. In: Kafatos, M., Ed., Bell $s$ Theorem, Quantum Theory and Conceptions of the Universe, Kluwer, Dordrecht, 69.

[52] Wallquist, M., Shumeiko, V.S. and Wendin, G. (2006) Physical Review B, 74, Article ID: 224506.

[53] Martinis, J.M., Cooper, K.B., McDermott, R., Steffen, M., Ansmann, M., Osborn, K.D., Cicak, K., Oh, S., Pappas, D.P., Simmonds, R.W. and Yu, C.C. (2005) Physical Review Letters, 95, Article ID: 210503.

https://doi.org/10.1103/PhysRevLett.95.210503 